\title{
Intervindo em atividade física, alimentação e estresse em moradores de município de pequeno porte
}

\author{
Intervention on physical activity, diet and stress in people living in a small city
}

\section{AUTORES \\ Leony Morgana Galliano ${ }^{1}$ (D) \\ Felipe Fossati Reichert ${ }^{1}$ (D) \\ 1 Universidade Federal de Pelotas, Programa de Pós-Graduação em Educação Física, Departamento de Educação Física, Pelotas, Rio Grande do Sul, Brasil}

\section{CONTATO}

Leony Morgana Galliano

Email:Imgalliano@gmail.com

Rua Luís de Camões, 625, Pelotas, Rio

Grande do Sul, Brasil.

CEP: $96055-630$

DOI

$10.12820 /$ rbafs. $24 \mathrm{e} 0097$

\begin{abstract}
RESUMO
Objetivou-se apresentar o modelo conceitual e desafios de uma intervenção de educação em saúde, incluída por meio do Sistema Único de Saúde. Todos moradores de Dona Francisca, Rio Grande do Sul, de 50-80 anos de idade, atendidos pelos oito agentes comunitários de saúde (ACS) foram considerados elegíveis para a intervenção, que abrangeu: i) encontro individual; ii) contatos motivacionais; iii) encontros educacionais relacionados à atividade física, alimentação e controle de estresse. A intervenção foi conduzida pelos ACS previamente treinados. Foram realizadas três coletas de dados: i) linha de base (2014; $n=649)$; ii) intermediária $(2015 ; n=544)$ e; iii) final $(2016 ; n=555)$. Os principais desafios foram relacionados a pactuação dos ACS, baixa participação nos encontros educacionais e coleta de dados, especialmente em áreas rurais. Porém, houve capacitação dos ACS, maior acesso às informações de saúde para a população e coletas de dados que possibilitam traçar o perfil de uma população de município de pequeno porte.
\end{abstract}

Palavras-chave: Educação em saúde; População rural; Participação da comunidade; Agentes comunitários de saúde; Atividade motora.

\begin{abstract}
The aim of this study is to present the rationale and challenges of a health education intervention, which occurred through the unified health system. All residents of Dona Francisca/Rio Grande do Sul, aged 50-80 years and serviced by community health workers $(C H W)$ were elegible for the intervention, which consisted of: i) individual meeting; ii) motivational contacts; iii) educational meetings related to physical activity, nutrition and stress control. The intervention was handled by $C H W$, previously trained. Data were collected for: i) baseline (2014; $n=649)$; ii) intermediate (2015; $n=544)$; and iii) final (2016; $n=555)$ periods. The main challenges were related to $C H W$ performance, low participantion in educational meetings and data collection, particulary among rural areas. However, there was training of $\mathrm{CHW}$, greater access to health information for the population and data collection that make it possible to know the profile of a population of a small city.
\end{abstract}

Keywords: Health education; Rural population; Community participation; Community health workers; Motor activity.
Este obra está licenciado com uma Licença Creative Commons Atribuição-NãoComercialCompartilhalgual 4.0 Internacional.

\section{Introdução}

O Sistema Único de Saúde (SUS) apresenta ações vinculadas à Atenção Primária à Saúde (APS), porta de entrada do usuário no sistema ${ }^{1}$. A estratégia da APS preconiza atendimentos com equipes multidisciplinares, alocadas nas Unidades Básicas de Saúde (UBS) e é guiada por eixos temáticos, entre eles, "práticas corporais/atividade física", fortalecendo a relevância de ações de promoção de atividades físicas nas comunidades.

Ações em promoção em saúde desenvolvidas por pessoas da comunidade, como os agentes comunitários em saúde (ACS), podem ser uma boa estratégia para aproximar o usuário do sistema devido a aspectos como eventual relação prévia, linguagem e interesses similares.
Considerando o aumento no número de idosos ${ }^{3}$ e das doenças crônicas ${ }^{4}$, intervenções para promoção de hábitos saudáveis vêm ganhando espaço, visando a manutenção da capacidade funcional, independência e melhoria na qualidade de vida ${ }^{5}$. Outro ponto que merece reflexão se refere ao fato de que majoritariamente a população brasileira reside em municípios de pequeno porte $^{3}$, sub-representados em pesquisas científicas.

Fundamentando-se por estes aspectos, elaborou-se intervenção em educação em saúde, com foco na atividade física, alimentação e controle do estresse, vinculada ao SUS. O presente estudo objetiva apresentar o modelo conceitual para a elaboração da ação, além dos desafios encontrados para sua implementação no município de Dona Francisca, Rio Grande do Sul. 


\section{Métodos}

Foi realizada uma intervenção em educação em saúde, entre 2014-2016, envolvendo adultos mais velhos (50-80 anos de idade), residentes em todo o território do município de Dona Francisca, Rio Grande do Sul (4 áreas urbanas e 4 rurais). Dona Francisca situa-se na região central do estado do Rio Grande do Sul e a principal atividade econômica é a agricultura. $\mathrm{O}$ município foi escolhido por haver uma relação prévia com a equipe de pesquisa. Dona Francisca tem cerca de 3000 habitantes $(33,7 \% \text { com idades entre } 50 \text { e } 80 \text { anos })^{3}$ e há apenas uma UBS para abranger toda a população. Todas as atividades da intervenção foram conduzidas pelos ACS - Estratégia Saúde da Família, treinados pela equipe de pesquisa (autora LMG).

Todos os residentes do município com idades entre 50-80 anos, atendidos pelos ACS foram convidados, excluindo-se aqueles com limitações físicas, cognitivas/ neurológicas que impossibilitassem responder o questionário ou participar da intervenção. Cada ACS listou os elegíveis em suas áreas, totalizando 758 pessoas.

Antes do início do estudo foram realizadas reuniões com os gestores municipais, os quais demonstraram interesse em desenvolver a intervenção. Após os contatos iniciais, realizaram-se encontros com todos os ACS do município $(\mathrm{n}=8)$ com explanação sobre as atividades propostas e acordos sobre os compromissos durante o processo. Neste momento, houve conformidade quanto à participação e execução das atividades propostas aos ACS. Além dos recursos humanos, a secretaria municipal da saúde se dispôs a ceder espaços físicos para as atividades e fornecer dados de saúde (prontuários) dos elegíveis.

Adotou-se a alocação aleatória dos ACS em dois grupos (A: intervenção em 2015 ou B: intervenção em 2016), com intuito de minimizar o compartilhamento de informações da intervenção entre grupos. Além disso, três áreas urbanas foram sorteadas para receber a intervenção no primeiro ano, prevendo a maior adesão dos residentes destas áreas, permitindo uma rápida avaliação. Assim, houve possibilidade de ajustes para o ano subsequente - que incluiu um número superior de áreas rurais.

O convite inicial para a intervenção ocorreu durante as visitas de rotina dos ACS e, mediante aceite, foi agendada a avaliação inicial.

\section{Avaliação quantitativa}

Cinco entrevistadores foram treinados presencialmente para realizarem entrevistas face-a-face no início do programa (2014), durante (2015) e após (2016). O treinamento incluiu leitura de manual/questionário, simulação de entrevistas, protocolo de avaliações físicas e acelerometria. A coleta foi realizada na residência do participante. O questionário incluiu dados sociodemográficos, saúde, comportamentais - atividade física, alimentação e estresse. Houve aferição de massa corporal, estatura, força isométrica de preensão manual.

Foi lido e explicado o termo de consentimento livre e esclarecido à todos os participantes. Em caso de ausência/recusa, foram realizadas três tentativas, em dias/ turnos e entrevistador diferente.

Treinamento dos Agentes Comunitários de Saúde Para o desenvolvimento de todas as ações, os ACS foram treinados pela equipe de pesquisa da Universidade Federal de Pelotas e University of Illinois at Urbana-Champaign (EUA). Durante o ano de 2015 os ACS do grupo A receberam o treinamento, enquanto o grupo B participou do treinamento em 2016. Os ACS do primeiro grupo foram orientados a não divulgar o conteúdo aos demais profissionais.

Foram realizados dez treinamentos, com duração aproximada de quatro horas/cada, contendo: descrição das atividades e simulação dos encontros educacionais. Os dois treinamentos iniciais abordaram temáticas introdutórias, o terceiro discorreu sobre o encontro individual e o seguinte sobre o contato motivacional - realizados antes da etapa de "mobilização e convite". Os treinamentos cinco a 10 foram realizados mensalmente e eram específicos sobre cada encontro educacional. As apostilas utilizadas foram elaboradas pela equipe de pesquisa das duas universidades (disponíveis: https:// osf.io/qxd8y/).

\section{Intervenção em educação em saúde}

A intervenção foi um programa educacional adaptado à cultura regional baseada em evidências prévias $^{6-12}$. Foram abordados temas de atividade física, alimentação e controle de estresse. O programa inclui um componente religioso, estratégia adotada devido à forte religiosidade entre idosos, o que hipotetiza-se que poderia aumentar a adesão, assimilação do conteúdo e, portanto, um maior interesse na intervenção. $O$ predomínio da religião católica, considerando a existência e inclusão das demais, é visível em Dona Francisca, Rio Grande do Sul, cidade com fortes raízes na colonização italiana e similar a muitas cidades pequenas brasileiras em que a igreja se localiza no centro da cidade. Segundo dados 
coletados no estudo, $89,0 \%$ da comunidade é católica praticante, $7,0 \%$ é evangélica, $2,17 \%$ pratica outras religiões (espírita, religiões afro-brasileira, testemunha de Jeová, protestante) e 1,83\% declararam não ter religião.
Este conteúdo foi desenvolvido após consultas à paróquia local para identificar histórias de santos que pudessem ser vinculadas aos temas. $\mathrm{O}$ quadro 1 apresenta o modelo lógico das atividades desenvolvidas.

Quadro 1 - Modelo lógico de atividades desenvolvidas no programa de educação em saúde em Saúde Dona Francisca, Rio Grande do Sul (2014-2016).

\begin{tabular}{|c|c|c|c|c|c|c|}
\hline Insumos & $\begin{array}{l}\text { Atividades } \\
\text { (momento) }\end{array}$ & Participantes & Estratégia & Participação & Produtos & Objetivos \\
\hline \multicolumn{7}{|l|}{ Implementadores } \\
\hline $\begin{array}{l}\text { UFPel, UIUC } \\
\text { SMS, Prefeitura Municipal } \\
\text { de Dona Francisca, Rio } \\
\text { Grande do Sul }\end{array}$ & $\begin{array}{l}\text { Convite inicial } \\
\text { (setembro - } \\
\text { outubro/2014) }\end{array}$ & Todos os elegíveis & $\begin{array}{l}\text { Visita domiciliar } \\
\text { realizada pelo ACS }\end{array}$ & $\mathrm{n}=758$ & & $\begin{array}{l}\text { Curto prazo: } \\
\text { Capacitação dos } \\
\text { ACS }\end{array}$ \\
\hline \multicolumn{7}{|l|}{ Recursos humanos } \\
\hline $\begin{array}{l}\text { ACS }(\mathrm{n}=8) \\
\text { Entrevistadores }(\mathrm{n}=5) \\
\text { Pesquisadores }(\mathrm{n}=4) \\
\text { Supervisão }(\mathrm{n}=1)\end{array}$ & $\begin{array}{l}\text { Coleta de dados } \\
\text { de linha de base } \\
\text { (outubro- } \\
\text { dezembro/2014) }\end{array}$ & Todos os elegíveis & $\begin{array}{l}\text { Entrevistas e } \\
\text { avaliações efetuados } \\
\text { na residência do } \\
\text { participante }\end{array}$ & $\mathrm{n}=649$ & $\begin{array}{l}\text { Questionários, } \\
\text { dados de } \\
\text { acelerometria, } \\
\text { avaliação física } \\
\text { (massa corporal, } \\
\text { estatura, força } \\
\text { isométrica de } \\
\text { preensão manual) }\end{array}$ & $\begin{array}{l}\text { Médio/longo prazo: } \\
\text { Mudanças } \\
\text { comportamentais } \\
\text { em atividade física, } \\
\text { alimentação e } \\
\text { controle do estresse }\end{array}$ \\
\hline \multicolumn{7}{|l|}{ Recursos físicos } \\
\hline $\begin{array}{l}\text { Sala dos ACS } \\
\text { Salão paroquial } \\
\text { Ginásios esportivos }\end{array}$ & $\begin{array}{l}\text { Randomização } \\
\text { considerando o } \\
\text { ACS de referência } \\
\text { (dezembro/2014) }\end{array}$ & & $\begin{array}{l}\text { Sorteio aleatório } \\
\text { realizado no } \\
\text { computador }\end{array}$ & & & \\
\hline \multicolumn{7}{|l|}{ Recursos materiais } \\
\hline $\begin{array}{l}\text { Questionários } \\
\text { Balanças digitais } \\
\text { Fita métrica } \\
\text { Dinamômetro de preensão } \\
\text { manual } \\
\text { Acelerômetros } \\
\text { Folhetos educativos } \\
\text { Material para determinação } \\
\text { de metas } \\
\text { Brindes } \\
\text { "Santinhos" (mini-folhetos } \\
\text { com imagem de um Santo } \\
\text { - conteúdo religioso) }\end{array}$ & $\begin{array}{l}\text { Treinamento dos } \\
\text { ACS } \\
\text { (frequência } \\
\text { mensal, entre } \\
\text { dezembro/2014 e } \\
\text { maio/2015) }\end{array}$ & $\begin{array}{l}4 \text { ACS do grupo } \\
\text { A (intervenção } \\
\text { em 2015) }\end{array}$ & $\begin{array}{l}\text { Atividade presencial } \\
\text { conduzida pela } \\
\text { supervisora } \\
\text { responsável. } \\
\text { Envolveu exposição } \\
\text { oral, leitura de } \\
\text { apostila e simulação } \\
\text { das atividades }\end{array}$ & $\mathrm{n}=4$ & $\begin{array}{l}\text { Apostilas } \\
\text { Apresentações de } \\
\text { slides }\end{array}$ & \\
\hline \multicolumn{7}{|l|}{ Recursos financeiros } \\
\hline \multirow{3}{*}{$\begin{array}{l}\text { Edital Pesquisador } \\
\text { Visitante - Ciências Sem } \\
\text { Fronteiras (2014) }\end{array}$} & $\begin{array}{l}\text { Encontro } \\
\text { individual para } \\
\text { estabelecimento } \\
\text { de metas } \\
\text { (fevereiro- } \\
\text { março/2015) }\end{array}$ & $\begin{array}{l}\text { Participantes do } \\
\text { grupo A }\end{array}$ & $\begin{array}{l}\text { Encontro domiciliar } \\
\text { realizado pelo ACS }\end{array}$ & $\mathrm{n}=389$ & $\begin{array}{l}\text { Material impresso } \\
\text { para estabelecimento } \\
\text { de metas e } \\
\text { com resultados } \\
\text { individualizados da } \\
\text { coleta inicial }\end{array}$ & \\
\hline & $\begin{array}{l}\text { Grupos } \\
\text { educacionais } \\
\text { (abril- } \\
\text { setembro/2015) }\end{array}$ & $\begin{array}{l}\text { Participantes do } \\
\text { grupo A }\end{array}$ & $\begin{array}{l}\text { Encontros em grupo } \\
\text { com atividades } \\
\text { expositivas, leituras, } \\
\text { atividades práticas } \\
\text { e componente } \\
\text { religioso }\end{array}$ & $\begin{array}{l}\mathrm{n}(\text { total })=150 \\
\text { Grupo } 1, \mathrm{n}=72 \\
\text { Grupo } 2, \mathrm{n}=101 \\
\text { Grupo } 3, \mathrm{n}=92 \\
\text { Grupo } 4, \mathrm{n}=88 \\
\text { Grupo } 5, \mathrm{n}=92 \\
\text { Grupo } 6, \mathrm{n}=76\end{array}$ & $\begin{array}{l}\text { Folhetos } \\
\text { educacionais } \\
\text { "Santinhos" } \\
\text { (conteúdo religioso) } \\
\text { Apresentação de } \\
\text { slides } \\
\text { Lista de frequência }\end{array}$ & \\
\hline & $\begin{array}{l}\text { Coleta } \\
\text { intermediária } \\
\text { (outubro- } \\
\text { dezembro/2015) }\end{array}$ & Todos os elegíveis & $\begin{array}{l}\text { Entrevistas e } \\
\text { avaliações efetuados } \\
\text { na residência do } \\
\text { participante }\end{array}$ & $\mathrm{n}=544$ & $\begin{array}{l}\text { Questionários, dados } \\
\text { de acelerometria, } \\
\text { avaliação física } \\
\text { (massa corporal, } \\
\text { estatura, força } \\
\text { isométrica de } \\
\text { preensão manual) }\end{array}$ & \\
\hline
\end{tabular}




\begin{tabular}{|c|c|c|c|c|c|c|}
\hline Insumos & $\begin{array}{l}\text { Atividades } \\
\text { (momento) }\end{array}$ & Participantes & Estratégia & Participação & Produtos & Objetivos \\
\hline \multirow{5}{*}{$\begin{array}{l}\text { Edital Pesquisador } \\
\text { Visitante - Ciências Sem } \\
\text { Fronteiras (2014) }\end{array}$} & $\begin{array}{l}\text { Treinamento dos } \\
\text { ACS } \\
\text { (frequência } \\
\text { mensal, entre } \\
\text { dezembro/2015 e } \\
\text { maio/2016) }\end{array}$ & $\begin{array}{l}4 \text { ACS do grupo } \\
\text { B (intervenção } \\
\text { em 2015) }\end{array}$ & $\begin{array}{l}\text { Atividade presencial } \\
\text { conduzida pela } \\
\text { supervisora } \\
\text { responsável. } \\
\text { Envolveu exposição } \\
\text { oral, leitura de } \\
\text { apostila e simulação } \\
\text { das atividades }\end{array}$ & $\mathrm{n}=4$ & $\begin{array}{l}\text { Apostilas } \\
\text { Apresentações de } \\
\text { slides }\end{array}$ & \\
\hline & $\begin{array}{l}\text { Encontro } \\
\text { individual para } \\
\text { estabelecimento } \\
\text { de metas } \\
\text { (fevereiro- } \\
\text { março/2016) }\end{array}$ & $\begin{array}{l}\text { Participantes do } \\
\text { grupo B }\end{array}$ & $\begin{array}{l}\text { Encontro domiciliar } \\
\text { realizado pelo ACS }\end{array}$ & $\mathrm{n}=310$ & $\begin{array}{l}\text { Material impresso } \\
\text { para estabelecimento } \\
\text { de metas e } \\
\text { com resultados } \\
\text { individualizados da } \\
\text { coleta intermediária }\end{array}$ & \\
\hline & $\begin{array}{l}\text { Grupos } \\
\text { educacionais } \\
\text { (abril- } \\
\text { setembro/2016) }\end{array}$ & $\begin{array}{l}\text { Participantes do } \\
\text { grupo B }\end{array}$ & $\begin{array}{l}\text { Encontros em grupo } \\
\text { com atividades } \\
\text { expositivas, leituras, } \\
\text { atividades práticas } \\
\text { e componente } \\
\text { religioso }\end{array}$ & $\begin{array}{l}\mathrm{n}(\text { total })=61 \\
\text { Grupo } 1, \mathrm{n}=49 \\
\text { Grupo } 2, \mathrm{n}=36 \\
\text { Grupo } 3, \mathrm{n}=17 \\
\text { Grupo } 4, \mathrm{n}=11 \\
\text { Grupo } 5, \mathrm{n}=25 \\
\text { Grupo } 6, \mathrm{n}=22\end{array}$ & $\begin{array}{l}\text { Folhetos } \\
\text { educacionais } \\
\text { "Santinhos" } \\
\text { (conteúdo religioso) } \\
\text { Apresentação de } \\
\text { slides } \\
\text { Lista de frequência }\end{array}$ & \\
\hline & $\begin{array}{l}\text { Coleta final } \\
\text { (outubro- } \\
\text { dezembro/2016) }\end{array}$ & Todos os elegíveis & $\begin{array}{l}\text { Entrevistas e } \\
\text { avaliações efetuados } \\
\text { na residência do } \\
\text { participante }\end{array}$ & $\mathrm{n}=555$ & $\begin{array}{l}\text { Questionários, } \\
\text { dados de } \\
\text { acelerometria, } \\
\text { avaliação física } \\
\text { (massa corporal, } \\
\text { estatura, força } \\
\text { isométrica de } \\
\text { preensão manual) }\end{array}$ & \\
\hline & $\begin{array}{l}\text { Encerramento } \\
\text { das atividades }\end{array}$ & $\begin{array}{l}\text { Todos os } \\
\text { elegíveis e equipe } \\
\text { de saúde do } \\
\text { município }\end{array}$ & $\begin{array}{l}\text { Confraternização } \\
\text { de encerramento } \\
\text { com divulgação dos } \\
\text { principais resultados }\end{array}$ & & & \\
\hline
\end{tabular}

Barreiras = acesso às áreas rurais para as coletas de dados; inicialmente houve desafios quanto à participação dos ACS nas atividades do estudo; adesão dos participantes das áreas rurais. Facilitadores = financiamento; comunicação entre a equipe de pesquisa e a de saúde; relação com a prefeitura e secretaria municipal de saúde; por se tratar de um município de pequeno porte, facilitou o alcance da intervenção na comunidade. UFPEL = Universidade Federal de Pelotas; UIUC = University of Illinois Urbana-Champaign; SMS = Secretaria Municipal de Saúde; ACS = Agente Comunitário de Saúde.

\section{Programa de educação em saúde}

A intervenção teve três eixos, sendo eles:

1) Encontro individual: Atividade realizada na residência do participante em que o ACS entregava um formulário impresso contendo: i) resultados individualizados da avaliação quantitativa anterior (atividade física, alimentação, índice de massa corporal) e; ii) plano de ação a médio/longo prazo: ACS conversava com o participante sobre estabelecimento de metas e auxiliava no preenchimento do formulário. Conforme o plano de ação, o ACS elaborava a estratégia de motivação individual.

2) Contatos motivacionais: Contatos telefônicos ou presenciais informais realizados semanalmente para o acompanhamento do progresso individual e suporte a eventuais dificuldades.
3) Encontros educacionais em grupo: Realizou-se seis encontros educacionais (um/mês) por grupos. Os grupos eram compostos por $\sim 50$ pessoas, com duração de $1 \mathrm{~h} 30 \mathrm{~min}$, havendo leituras relacionadas ao tema (benefícios, formas práticas de inserção do comportamento na rotina - 30min), atividades práticas (demonstrações de preparos de alimentos, atividades físicas e práticas de relaxamento - 40min), distribuição de brindes e conteúdo religioso (história de um santo e entrega de mini-folheto - popularmente conhecido como "santinho" - com a imagem do santo correspondente - 20min) - Quadro 2. Em cada encontro foi entregue um livreto educacional apresentando informações sobre o tema abordado.

O material educacional foi desenvolvido pela equipe de pesquisa, sendo que o componente de atividade 
Quadro 2 - Descrição dos componentes abordados durante os encontros educacionais, Estudo de Saúde Dona Francisca, 2014-2016.

\begin{tabular}{|c|c|c|c|c|}
\hline Título & Tema da leitura & Atividade prática & Brinde & Conteúdo religioso \\
\hline $\begin{array}{l}\text { Energize-se com uma } \\
\text { alimentação saudável }\end{array}$ & $\begin{array}{l}\text { Benefícios e formas de inserir } \\
\text { frutas, verduras, alimentos } \\
\text { integrais e água na rotina } \\
\text { alimentar. Consumo de açúcar }\end{array}$ & $\begin{array}{l}\text { Pesar porções de frutas e verduras; } \\
\text { Demonstração da quantidade de açúcar } \\
\text { em alimentos e bebidas (exemplo: } \\
\text { brigadeiro, refrigerante) }\end{array}$ & Sacola retornável & Santo Antônio \\
\hline Viva ativo a sua maneira & $\begin{array}{l}\text { Benefícios e como realizar } \\
\text { atividades físicas aeróbias no dia- } \\
\text { a-dia (duração e intensidade) }\end{array}$ & $\begin{array}{l}\text { Caminhada na praça com uso do } \\
\text { pedômetro }\end{array}$ & Pedômetro & Papa João Paulo II \\
\hline De bem com a vida & $\begin{array}{l}\text { Como se alimentar com atenção } \\
\text { plena nas refeições? Como } \\
\text { manejar o estresse e o quanto } \\
\text { pode impactar nas refeições? }\end{array}$ & $\begin{array}{l}\text { Prática de atenção plena enquanto se } \\
\text { alimenta. Comer uva-passa atento ao tato, } \\
\text { visão, olfato, paladar }\end{array}$ & $\begin{array}{l}\text { CD de meditação e } \\
\text { pulseira do bem querer }\end{array}$ & Santo Padre Pio \\
\hline $\begin{array}{l}\text { Preparando refeições mais } \\
\text { saudáveis }\end{array}$ & $\begin{array}{l}\text { Quantidades recomendadas e } \\
\text { efeitos do consumo de sal. Tipos } \\
\text { de gordura e como interferem na } \\
\text { nossa saúde }\end{array}$ & $\begin{array}{l}\text { Leitura de rótulos - identificar } \\
\text { quantidade de gordura e sal nos alimentos } \\
\text { (exemplo: tipos de leites, conservas) }\end{array}$ & Copo plástico & $\begin{array}{l}\text { Nossa Senhora da } \\
\text { Saúde }\end{array}$ \\
\hline Forte para a vida & $\begin{array}{l}\text { Como incluir atividades físicas de } \\
\text { resistência muscular no dia-a-dia }\end{array}$ & $\begin{array}{l}\text { Demonstração de exercícios de força e } \\
\text { resistência muscular utilizando faixas elásticas }\end{array}$ & $\begin{array}{l}\text { Faixas elásticas (thera } \\
\text { band) }\end{array}$ & São Francisco \\
\hline $\begin{array}{l}\text { Melhorando sua qualidade } \\
\text { de vida }\end{array}$ & $\begin{array}{l}\text { Como realizar atividade física } \\
\text { com atenção plena? Como a } \\
\text { atividade física pode auxiliar no } \\
\text { controle do estresse }\end{array}$ & $\begin{array}{l}\text { Realização de caminhada com atenção } \\
\text { plena (observando o trajeto, árvores, canto } \\
\text { dos pássaros) }\end{array}$ & $\begin{array}{l}\text { Pulseira dia do } \\
\text { pensamento positivo }\end{array}$ & Padre Manuel \\
\hline
\end{tabular}

física foi baseado nas diretrizes do American College of Sports Medicine $e^{13}$ (livretos disponíveis: http://adl.kch. illinois.edu/healthy-lifestyle\#toverlay-context=content/dona-francisca-health-study).

\section{Avaliação do programa}

Como avaliação do programa foi utilizado o modelo RE-AIM ${ }^{14}$, ferramenta translacional que aproxima pesquisa e contexto real. O modelo foi utilizado para avaliar o programa nos níveis individuais e organizacionais através de entrevistas qualitativas semiestruturadas. Foram entrevistados 37 elegíveis escolhidos pelos ACS. Os participantes foram selecionados conforme diferentes níveis de frequência nas atividades, contemplando aqueles que estiveram presentes em todas as atividades, ausentes em todas as atividades e aqueles que participaram de algumas atividades. Além disso, foram entrevistados, antes e após a intervenção: prefeito, vice-prefeita, secretária municipal de saúde, padres, gestora da UBS, profissionais da equipe de saúde e todos os ACS.

\section{Logística para coleta de dados}

Para localizar os participantes das áreas urbanas os entrevistadores recebiam mapas, endereços, pontos de referência e apelidos dos participantes. Para as entrevistas no meio rural, a prefeitura disponibilizou o transporte, sendo acompanhado pelo ACS responsável pela área. Para o presente trabalho, consideramos como resulta- dos a percepção da equipe de pesquisa sobre os desafios e pontos fortes encontrados durante a implementação e intervenção.

\section{Apontamentos sobre dificuldades e potências}

a) Pactuação com a equipe: Todos os ACS indicaram aceite quanto as tarefas que seriam desenvolvidas, desde que estivessem dentro dos seus horários de trabalho. Porém, no decorrer do campo e intervenção foram encontradas dificuldades na adesão por parte dos ACS, sendo que em contato com participantes da intervenção, foram identificadas ausência de convites para a coleta de dados e grupos educacionais. Por outro lado, após reuniões e durante os treinamentos foi possível estabelecer um relacionamento proximal, aumentando a pactuação. Durante a capacitação buscou-se promover o entendimento do papel central que os ACS tinham para o sucesso da intervenção, podendo impactar positivamente na saúde da comunidade. Além de disponibilizar os materiais para a intervenção, foram doados equipamentos eletrônicos para a UBS (tablet, notebook) e, ao término da última coleta de dados, os ACS receberam da equipe de pesquisa uma gratificação pela participação (R\$ 300,00/cada). Em nenhum momento os ACS estavam cientes que receberiam qualquer recompensa pelo trabalho.

b) Encontros individuais: Para este componente, a prin- 
cipal dificuldade apontada pelos ACS se referiu ao entendimento de parte dos participantes quanto ao estabelecimento de metas e elaboração do plano de ação para mudança de comportamento. Neste momento os participantes não haviam recebido materiais educacionais e participado de encontros, o que poderia contribuir para a elaboração de metas pessoais.

Ressalta-se que essas dificuldades ocorreram especialmente entre pessoas com baixa escolaridade. Em contrapartida, verificou-se que entre as pessoas que aderiram à intervenção, essa etapa foi fundamental para despertar o interesse nas temáticas abordadas, além de centralizar no indivíduo o estabelecimento e formas de cumprirem suas metas.

c) Contatos motivacionais: Para alguns participantes da área rural este componente não ocorreu com a frequência estipulada em virtude de indisponibilidade de cobertura telefônica e dificuldade de acesso à residência para realizar o contato pessoalmente. Nas áreas urbanas, na maioria dos casos, os ACS optaram por realizar o contato presencialmente, já que as residências são próximas e as visitas são rotineiras. Os contatos motivacionais tiveram um papel importante para manter a adesão e acompanhar os progressos/barreiras dos participantes, além da obtenção de um feedback para que os ACS se preparassem para os encontros subsequentes.

d) Encontros em grupo: As maiores dificuldades apontadas pelos participantes se relacionaram aos horários disponibilizados (manhã/tarde), o que impossibilitou a participação daqueles que trabalhavam durante o dia. Além disso, alguns moradores das áreas rurais indicaram dificuldades de acesso, apesar de serem escolhidos pontos estratégicos. Porém, as características das localidades (estrada, morros) e a necessidade de veículo para deslocamento, podem ter impactado na participação. No início do estudo, houve uma preocupação da equipe de pesquisa quanto a aceitação do conteúdo religioso, especialmente entre os não-católicos (11,0\%). Por este motivo, pediu-se aos ACS que este componente fosse o último a ser realizado nos encontros, dando a opção aos participantes de presenciar ou não a atividade. Porém, segundo relato dos ACS e dos participantes, o conteúdo religioso foi bem aceito entre todos (praticantes ou não de religiões). Este componente foi estruturado para que, aliado aos conteúdos de saúde, pudesse ter um papel importante para mudanças de estilo de vida. Contudo, caso fosse ob- servada alguma rejeição por parte dos participantes, poderia ter sido excluído do programa sem gerar prejuízos, mas não foi necessário. Contrapondo, considerando que cerca de metade dos participantes exerciam tarefas remuneradas e grande parte residia em áreas rurais $(40,0 \%)$, acredita-se que os encontros tiveram uma boa participação. A média de frequência foi de 3,16 \pm 1,77 encontros, 12,3\% participaram de todas as atividades propostas e $27,0 \%$ foram em um encontro.

e) Coleta de dados: Foram encontradas dificuldades logísticas para a coleta de dados nas áreas rurais, como: impossibilidade de acesso com veículos, enchentes, queda de pontes, não localização dos participantes (trabalho no campo). Alguns problemas foram contornados com ajuda dos ACS, que conhecem os trajetos mais seguros, além de identificarem os melhores horários para localizar os participantes, inclusive nas lavouras para agendamento de entrevista. Nas áreas urbanas, ainda no primeiro ano, observou-se que grande parte dos participantes era conhecida apenas por apelidos e a numeração das casas não era sequencial. Porém, essas situações foram resolvidas rapidamente conversando com os ACS e solicitando maiores detalhes sobre cada pessoa. Ao total, entre os 758 elegíveis, 699 pessoas foram entrevistadas ao menos uma vez ao longo dos três anos (2014; $\mathrm{n}=649.2015, \mathrm{n}=544 ; 2016, \mathrm{n}=$ 555), atingindo um percentual de $92,2 \%$ de cobertura. As maiores proporções de perdas e recusas foram de homens $(21,1 \%)$ e pessoas com idades entre $50-60$ anos $(34,1 \%)$.

A partir da intervenção proposta, foi possível inserir ações em educação em saúde no município de Dona Francisca, Rio Grande do Sul, vinculado ao SUS. A ação incluiu avaliações em saúde, além de programa educacional com conteúdos relacionados à alimentação, atividade física e controle de estresse. As maiores dificuldades foram relacionadas a atuação dos ACS, baixa participação nos encontros educacionais e coleta de dados nas áreas rurais. Por outro lado, houve capacitação dos ACS, maior oportunidade de acesso às informações de saúde para a população, além de haver coletas de dados que possibilitam traçar o perfil de uma população de município de pequeno porte.

\section{Conflito de interesse}

Os autores declaram não haver conflito de interesse. 


\section{Contribuição dos autores}

Todos os autores participaram da concepção do estudo. Galliano LM, foi responsável pela redação do texto e coleta de dados. Reichert FF, supervisionou todas as etapas do estudo. Ambos os autores revisaram o texto criticamente e aprovaram a versão final.

\section{Agradecimentos}

Ao financiamento proveniente do Edital do Programa Ciência sem Fronteiras - Chamada de Projetos no 09/2014 - 1o Cronograma - Pesquisador Visitante Especial.

\section{Referências}

1. Brasil. Ministério da Saúde. Secretaria de Vigilância em Saúde. Política nacional de promoção da saúde/Ministério da Saúde, Secretaria de Atenção à Saúde. - Brasília: Ministério da Saúde, 2006.

2. Brasil. Ministério da Saúde. Secretaria de Vigilância em Saúde. Secretaria de Atenção à Saúde. Política Nacional de Promoção da Saúde: PNPS: Anexo I da Portaria de Consolidação no 2 , de 28 de setembro de 2017, que consolida as normas sobre as políticas nacionais de saúde do SUS/ Ministério da Saúde, Secretaria de Vigilância em Saúde, Secretaria de Atenção à Saúde. - Brasília: Ministério da Saúde, 2018.

3. Instituto Brasileiro de Geografia e Estatísticas (IBGE). Síntese de indicadores sociais: uma análise das condições de vida da população brasileira. Rio de Janeiro: 2010.

4. Schmidt MI, Duncan BB, Azevedo e Silva G, Menezes AM, Monteiro CA, Barreto SM, et al. Chronic non-communicable diseases in Brazil: burden and current challenges. Lancet. 2011;377(9781):1949-61.

5. Chodzko-Zajko WJ, Schwingel A, Park CH. Successful aging: the role of physical activity. Am J Lifestyle Med. 2009;3:20-8.

6. Arandia G, Nalty C, Sharkey JR, Dean WR. Diet and acculturation among hispanic/latino older adults in the United States: a review of literature and recommendations. J Nutri Gerontol Geriatr. 2012;31(1):16-37.
7. Broyles SL, Brennan JJ, Herzog K, Kozo J, Taras HL. Cultural adaptation of a nutrition education curriculum for latino families to promote acceptance. J Nutrition Educ Behav. 2011;43(4 Suppl 2):S158-S61.

8. Frable PJ, Dart L, Bradley PJ. Peer Reviewed: Healthy weight (el camino saludable) phase 1: a retrospective critical examination of program evaluation. Prev Chronic Dis. 2006;3(3):A8.

9. James KS, Connelly CD, Rutkowski E, McPherson D, Gracia L, Mareno N, et al. Family-based weight management with latino mothers and children. J Spec Pediatr Nurs. 2008;13(4):249-62.

10. Olvera N, Bush JA, Sharma SV, Knox BB, Scherer RL, et al. BOUNCE: A community-based mother-daughter healthy lifestyle intervention for low-income latino families. Obesity. 2010;18(Suppl 1):S102:S104.

11. Healthier U.S. Gov. Be active your way: a guide for adults. 2008. [citado em 2019 fev 10]. Disponível em: https://health. gov/sites/default/files/2019-09/paguide.pdf.

12. Healthier U.S. Gov. Mantengase activo a su manera. Guia para adultos. 2008. [citado em 2019 jan 20]. Disponível em: https://health.gov/paguidelines/2008/pdf/PAG_Spanish_ Booklet.pdf.

13. Haskell WL, Lee IM, Pate RR, Powell KE, Blair SN, Franklin BA, et al. Physical activity and public health: updated recommendation for adults from the American College of Sports Medicine and the American Heart Association. Med Sci Sports Exerc. 2007;39(8):1423-34.

14. King DK, Glasgow RE, Leeman-Castillo B. Reaiming REAIM: using the model to plan, implement, and evaluate the effects of the environmental change approaches to enhancing population health. Am J Public Health. 2010;100(11):2076-84.

Recebido: $14 / 02 / 2019$ Aprovado: 26/03/2020 